\title{
Sensitivity of direct detection experiments to neutrino magnetic dipole moments
}

\author{
D. Aristizabal Sierra, ${ }^{a, b}$ R. Branada, ${ }^{a}$ O.G. Miranda ${ }^{c}$ and G. Sanchez Garcia ${ }^{c}$ \\ ${ }^{a}$ Universidad Técnica Federico Santa María - Departamento de Física, \\ Casilla 110-V, Avda. España 1680, Valparaíso, Chile \\ ${ }^{b}$ IFPA, Dep. AGO, Université de Liège, \\ Bat B5, Sart Tilman B-4000 Liège 1, Belgium \\ ${ }^{c}$ Departamento de Física, Centro de Investigación y de Estudios Avanzados del IPN, \\ Apartado Postal 14-740 07000 Mexico, Distrito Federal, Mexico \\ E-mail: daristizabal@ulg.ac.be, rocio.branada@sansano.usm.cl, \\ omr@fis.cinvestav.mx, gsanchez@fis.cinvestav.mx
}

ABSTRACT: With large active volume sizes dark matter direct detection experiments are sensitive to solar neutrino fluxes. Nuclear recoil signals are induced by ${ }^{8} \mathrm{~B}$ neutrinos, while electron recoils are mainly generated by the pp flux. Measurements of both processes offer an opportunity to test neutrino properties at low thresholds with fairly low backgrounds. In this paper we study the sensitivity of these experiments to neutrino magnetic dipole moments assuming 1, 10 and 40 tonne active volumes (representative of XENON1T, XENONnT and DARWIN), $0.3 \mathrm{keV}$ and $1 \mathrm{keV}$ thresholds. We show that with nuclear recoil measurements alone a 40 tonne detector could be as competitive as Borexino, TEXONO and GEMMA, with sensitivities of order $8.0 \times 10^{-11} \mu_{B}$ at the $90 \%$ CL after one year of data taking. Electron recoil measurements will increase sensitivities way below these values allowing to test regions not excluded by astrophysical arguments. Using electron recoil data and depending on performance, the same detector will be able to explore values down to $4.0 \times 10^{-12} \mu_{B}$ at the $90 \%$ CL in one year of data taking. By assuming a 200-tonne liquid xenon detector operating during 10 years, we conclude that sensitivities in this type of detectors will be of order $10^{-12} \mu_{B}$. Reducing statistical uncertainties may enable improving sensitivities below these values.

Keywords: Beyond Standard Model, Neutrino Physics, Solar and Atmospheric Neutrinos

ArXiv EPRINT: 2008.05080 


\section{Contents}

$2 \mathrm{CE} \nu \mathrm{NS}, \nu-e$ elastic scattering and neutrino magnetic dipole moments 2

2.1 Neutrino magnetic/electric dipole moments and cross sections 3

2.2 Recoil spectra 4

3 Sensitivity to neutrino magnetic moments $\quad 6$

4 Conclusions

\section{Introduction}

Dark matter $(\mathrm{DM})$ direct detection experiments are already sensitive to solar neutrinos. In its latest data sets, XENON1T has reported signals in both coherent elastic neutrinonucleus scattering ( $\mathrm{CE} \nu \mathrm{NS})$ and neutrino-electron elastic scattering [1, 2]. It is natural to expect that with increasing active volumes and exposures, XENONnT [3], LZ [4], and DARWIN [5] will provide larger statistics in both channels. Threfore, their results will enable precise measurements of neutrino properties complementing those coming from present and near-future dedicated neutrino experiments (see, e.g. [6-11]). The opportunities these data offer include - but are not limited to - studies of new interactions in the neutrino sector by means of light vector and scalar mediators, neutrino non-standard interactions, and neutrino electromagnetic properties [12-20]. They will also provide a playground for precise measurements of solar neutrino fluxes, including those from the solar CNO cycle [21], as well as for tests of solar models and solar neutrino matter effects [22].

With precise discrimination, measurements of electron or nuclear recoils alone can determine the presence of new physics. That could be the case - for instance - of the recent electron excess reported by the XENON1T collaboration [2], if indeed new physics is responsible for such a signal. Ideally, a physical explanation of an electron excess would produce a particular signature in the corresponding nuclear channel. However, an observation of a signal e.g. in electron recoils does not necessarily implies an observation of nuclear recoils. The main reason being the energy thresholds involved. For $\sim 0.1 \mathrm{keV}$ thresholds, electron recoils are driven by pp neutrino fluxes, while nuclear recoils by ${ }^{8} \mathrm{~B}$ neutrinos. In $\mathrm{cm}^{-2} \mathrm{~s}^{-1}$ units, these fluxes differ by about four orders of magnitude [23]. Thus, unless the new physics effects are way more pronounced in the $\nu-N$ cross section, one expects the electron recoil signal to be more prominent (possibly only observable in that channel). An extreme case of such scenario would be a nucleon-phobic light vector mediator, in which case only an excess in electron recoils could be observed. 
However, there are other cases in which either the electron or nuclear recoil signal can come along with a signal in the other channel. Arguably the most remarkable example in this case is given by photon exchange. As soon as the new physics couples to photons, depending on the size of the new physics couplings, there will be new contributions to electron and nuclear recoil signals. ${ }^{1}$ In the case of neutrino detection, any of its electromagnetic couplings will lead to both electron and nuclear recoils, again depending on their size both processes could be observed. This discussion is of course not only related to neutrinos, a typical example involving DM is given by dark photon portals in which kinetic mixing allows coupling of the dark and visible (SM) sectors [25, 26].

Motivated by the latest XENON1T result [2], in this paper we study the extent at which neutrino electromagnetic properties can be tested at XENON1T, XENONnT and DARWIN using combined electron and nuclear recoil measurements. We consider neutrino magnetic dipole moments and determine the discovery reach under simplified detector and signal assumptions. This includes one, ten and forty tonne active volumes, $100 \%$ detector efficiency and $0.3 \mathrm{keV}$ and $1 \mathrm{keV}$ energy thresholds. The former motivated by ref. [27], while the latter determined by future detector performances [3-5]. In all cases our toy experiments correspond to the SM electron and nuclear recoil spectra (measured in events/tonne/year/keV). For CE $\nu$ NS we assume two background hypotheses, $68 \%$ and $25 \%$ of the signal rate. Note that this choice is not intended to be understood as representative of what the actual backgrounds will be. The main motivation for this choice is that of showing the impact on sensitivities that background will have. And so to emphasize the importance of a proper understanding of the different backgrounds these experiments will be subject to. For $\nu-e$ elastic scattering we instead use expected backgrounds at XENON1T, XENONnT and DARWIN as given in refs. [2, 22, 28]. Needless to say, these assumptions - in particular for $\mathrm{CE} \nu \mathrm{NS}$ - are just representative of how the actual detectors performances and data sets will look like, but allow us to visualize how competitive these detectors will be when compared to neutrino dedicated experiments.

The remainder of this paper is organized as follows. In section 2 we shortly discuss $\mathrm{CE} \nu \mathrm{NS}, \nu-e$ elastic scattering as well as neutrino magnetic dipole moments and their corresponding differential cross sections. In section 3 we describe our statistical analysis and present our results. Finally in section 4 we summarize and present our conclusions.

\section{$2 \mathrm{CE} \nu \mathrm{NS}, \nu-e$ elastic scattering and neutrino magnetic dipole moments}

In the $\mathrm{SM}, \mathrm{CE} \nu \mathrm{NS}$ is a neutral current process in which the neutrino energy involved, $E_{\nu} \lesssim 100 \mathrm{MeV}$, is such that the transferred momentum implies the individual nucleon amplitudes sum up coherently. This results in an approximately overall enhancement of the cross section, determined by $N^{2}$, where $N$ refers to the number of neutrons of the target nuclei $[29,30]$. Given the constraints over the neutrino energy probe, possible neutrinos that can induce the process are limited to neutrinos produced in pion decay-at-rest, reactor

\footnotetext{
${ }^{1}$ Note that even in the most extreme case, nucleon- or lepto-phobic interactions, reconstruction of the signal will require electron and nuclear recoil measurements. For neutrino nuclear recoil traces of leptophobic scenarios see $[18,24]$.
} 
and solar neutrinos. Other possible sources include sub-GeV atmospheric neutrinos, diffuse supernova (SN) background (DSNB) neutrinos and a SN burst. However, their detection is less certain. Sub-GeV atmospheric neutrinos and DSNB fluxes are small, and so very large exposures are required for their detection [31]. Observation of a SN burst is not guaranteed, although it is expected to happen at certain point [32].

The differential cross section for this process involves a zero-transferred momentum component and a nuclear form factor that accounts for nuclear structure. It is given by [29]

$$
\frac{d \sigma_{\nu-N}}{d E_{r}}=\frac{G_{F}^{2}}{2 \pi} m_{N} g_{N}^{2}\left(2-\frac{m_{N} E_{r}}{E_{\nu}^{2}}\right) F^{2}\left(E_{r}\right) .
$$

Here $g_{N}=(A-Z) g_{N}^{n}+Z g_{V}^{p}$, where $g_{N}^{n}=2 g_{N}^{d}+g_{N}^{u}$ and $g_{N}^{p}=g_{N}^{d}+2 g_{N}^{u}$ with the quark electroweak couplings given by $g_{N}^{d}=-1 / 2+2 / 3 \sin ^{2} \theta_{W}$ and $g_{N}^{u}=1 / 2-4 / 3 \sin ^{2} \theta_{W}$. For the weak mixing angle we use $\sin ^{2} \theta_{W}=0.23122$ [33]. For the nuclear form factor we adopt the Helm parametrization and assume the same root-mean-square radii for the proton and neutron distributions. Assuming otherwise requires weighting the neutron and proton contributions with independent form factors [34]. Note that for the energies we are interested in the form factor plays a somewhat minor role.

Solar neutrinos are subject to neutrino flavor conversion, which depending on the process of the pp chain they originate from can be matter enhanced. Assuming the twoflavor approximation (mass dominance limit $\Delta m_{13}^{2} \rightarrow \infty$ ), two neutrino flavors reach the detector. Neutrino-electron scattering induced by solar neutrinos receives therefore contributions from neutral and charged current. Neutral from $\nu_{e}-e$ and $\nu_{a}-e$ interactions, while charged from $\nu_{e}-e$ alone. The differential cross section reads [35]

$$
\frac{d \sigma_{\nu-e}}{d E_{r}}=\frac{G_{F}^{2} m_{e}}{2 \pi}\left[\left(g_{V}+g_{A}\right)^{2}+\left(g_{V}-g_{A}\right)^{2}\left(1-\frac{E_{r}}{E_{\nu}}\right)^{2}\left(g_{A}^{2}-g_{V}^{2}\right) \frac{m_{e} E_{r}}{E_{\nu}^{2}}\right],
$$

with the vector and axial couplings given by

$$
g_{V}=2 \sin ^{2} \theta_{W} \pm \frac{1}{2}, \quad g_{A}= \pm \frac{1}{2} .
$$

Here ' + ' holds for $\nu_{e}$, while '-' for $\nu_{a}$.

\subsection{Neutrino magnetic/electric dipole moments and cross sections}

Possible neutrino electromagnetic couplings are determined by the neutrino electromagnetic current, which decomposed in terms of electromagnetic form factors leads to four diagonal independent couplings in the zero-transferred momentum limit: electric charge, magnetic dipole moment [36-41], electric dipole moment and anapole moment [39]. Depending on whether neutrinos are Dirac or Majorana and on whether CP and CPT are exact symmetries in the new physics sector, some of these couplings may vanish $[39,40]$. They are subject to a variety of limits from laboratory experiments [42] that include PVLAS [43], neutron $\beta$ decay [44], TRISTAN, LEP and CHARM-II [45], MUNU [46], Super-Kamiokande [47], TEXONO [6], GEMMA [48] and Borexino Phase-II [49]. They are constrained by cosmology and astrophysical observations as well, including primordial nucleosynthesis [50], 
neutrino star turning [51], supernova and stellar cooling [52-55]. For an extensive review on this constraints see ref. [56] (see ref. [57] as well for constraints involving order $\mathrm{MeV}$ right-handed neutrinos). ${ }^{2}$

At the effective level the magnetic/dipole couplings can be written according to

$$
\begin{aligned}
-\mathcal{L}^{D} & =\frac{1}{2} \overline{\nu_{L_{i}}} \sigma_{\mu \nu}\left(\mu_{i j}^{D}+\gamma_{5} \epsilon_{i j}^{D}\right) \nu_{R_{j}}+\text { H.c. } \\
-\mathcal{L}^{M} & =\frac{1}{2} \overline{\nu_{L_{i}}^{c}} \sigma_{\mu \nu}\left(\mu_{i j}^{M}+\gamma_{5} \epsilon_{i j}^{M}\right) \nu_{L_{j}}+\text { H.c. }
\end{aligned}
$$

Note that electron recoil experiments cannot differentiate between Dirac or Majorana couplings, nor between magnetic/electric moments or transitions. In the mass eigenstate basis, processes induced by interactions (2.4) are sensitive to the effective parameter [60]

$$
\mu_{\nu_{\mathrm{eff}}}^{2}=\sum_{j}\left|\sum_{k} A_{k}\left(E_{\nu}, L\right)\left(\mu_{k j}-i \epsilon_{k j}\right)\right|^{2},
$$

where $A_{k}\left(E_{\nu}, L\right)$ refers to the amplitude of the $k$-th massive neutrino state at detection point. The effective coupling takes different forms depending on whether neutrinos are Dirac or Majorana as well as on the flavor scheme adopted (see e.g. ref. [42]). We will therefore use this effective coupling in our calculations since it is useful from the phenomenological point of view, given that the analysis of neutrino magnetic interactions is in general - a multiparameter problem that can be reduced to a single parameter problem with the aid of (2.5). Using this parametrization, the $\nu-e$ differential cross section reads [35]

$$
\frac{d \sigma_{\nu}}{d E_{r}}=\pi \alpha \frac{\mu_{\nu_{\mathrm{eff}}}^{2}}{m_{e}^{2}}\left(\frac{E_{\nu}-E_{r}}{E_{\nu} E_{r}}\right)
$$

where $\mu_{\nu_{\text {eff }}}$ has been normalized to the Bohr magneton. For CE $\nu$ NS the differential cross section has the same structure but comes along with the number of target protons squared $Z^{2}$ and a nuclear form factor. Because of the Coulomb divergence the cross section is forward peaked, a behavior that becomes rather pronounced at low recoil energies. The most salient feature of neutrino magnetic moment interactions is thus spectral distortions.

For all the cross sections we have discussed maximum recoil energies are written as

$$
\begin{array}{ll}
\text { Neutrino-electron : } & E_{r}^{\max }=\frac{2 E_{\nu}^{2}}{m_{e}+2 E_{\nu}}, \\
\text { Neutrino-nucleus : } & E_{r}^{\max }=\frac{2 E_{\nu}^{2}}{m_{N}+2 E_{\nu}} \simeq \frac{2 E_{\nu}^{2}}{m_{N}},
\end{array}
$$

with the approximation being fairly good for all isotopes of interest, particularly for xenon.

\section{$2.2 \quad$ Recoil spectra}

The recoil spectrum for nuclear and electron recoils proceeds from a convolution of neutrino fluxes and neutrino cross sections. In the case of nuclear recoils, they will be sensitive to all

\footnotetext{
${ }^{2}$ For constraints on neutrino magnetic moments using Borexino data see e.g. [58]. For constraints on light vector mediators using TEXONO, CHARM-II and GEMMA see e.g. [59].
} 


\begin{tabular}{|c|c|}
\hline Component & Kinematic limit $[\mathrm{keV}]$ \\
\hline $\mathrm{pp}$ & $2.64 \times 10^{2}$ \\
\hline${ }^{7} \mathrm{Be}\left(E_{\nu}=0.3 \mathrm{MeV}\right)$ & $2.31 \times 10^{2}$ \\
\hline${ }^{7} \mathrm{Be}\left(E_{\nu}=0.8 \mathrm{MeV}\right)$ & $6.64 \times 10^{2}$ \\
\hline pep & $1.18 \times 10^{3}$ \\
\hline hep & $1.85 \times 10^{4}$ \\
\hline${ }^{8} \mathrm{~B}$ & $1.63 \times 10^{4}(4.48)$ \\
\hline${ }^{13} \mathrm{~N}$ & $9.88 \times 10^{2}$ \\
\hline${ }^{15} \mathrm{O}$ & $1.51 \times 10^{3}$ \\
\hline${ }^{17} \mathrm{~F}$ & $1.52 \times 10^{3}$ \\
\hline
\end{tabular}

Table 1. Kinematic recoil energy limit for the different neutrino components of the solar pp and $\mathrm{CNO}$ cycles in neutrino-electron scattering. Included as well in parenthesis is the kinematic limit for ${ }^{8} \mathrm{~B}$ in $\mathrm{CE} \nu \mathrm{NS}$. The values displayed follow from the BS05 standard solar model [23].
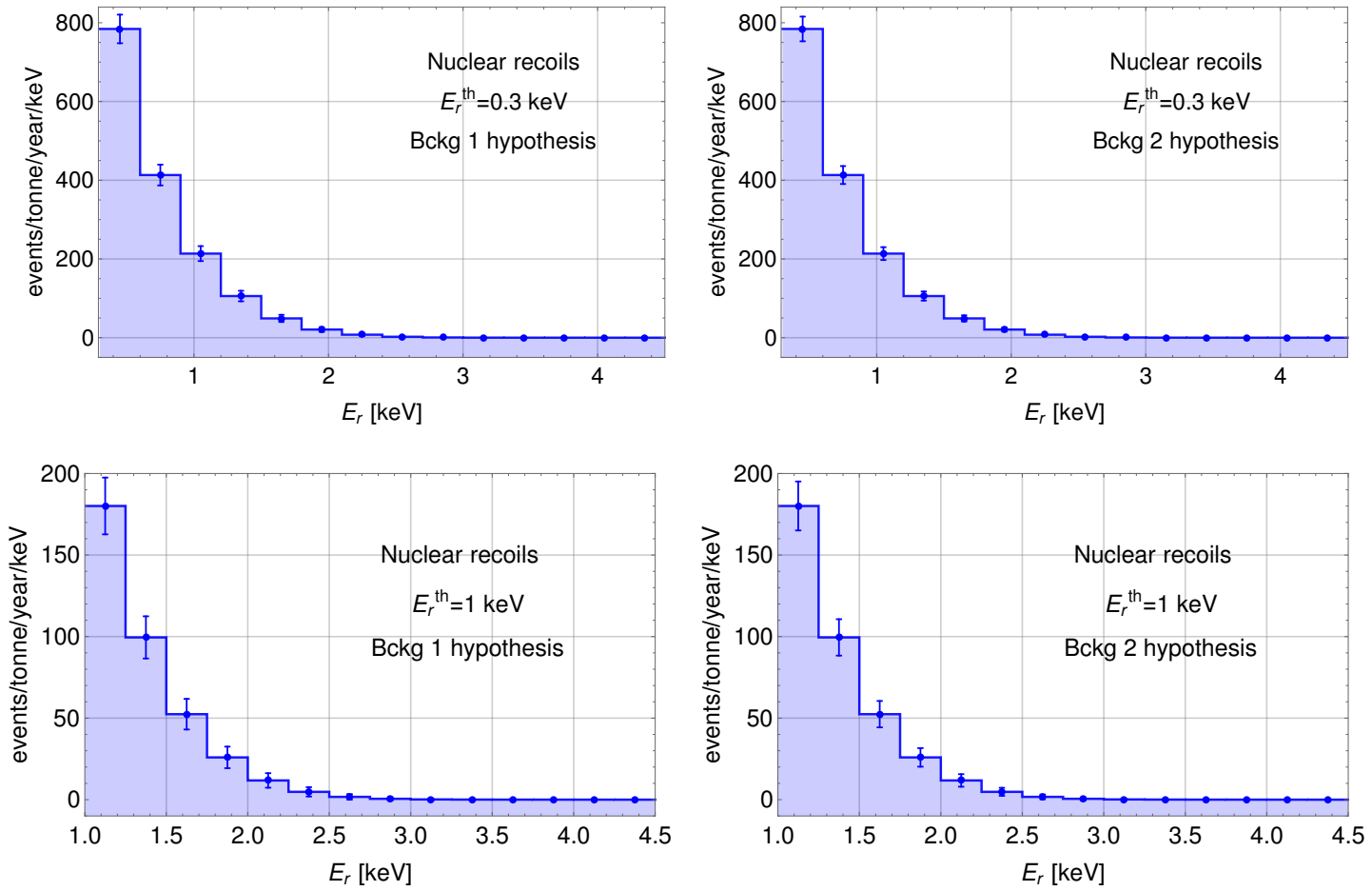

Figure 1. Top graphs: $\mathrm{CE} \nu \mathrm{NS}$ toy experiment signals for a $0.3 \mathrm{keV}$ threshold with a background amounting to $68 \%$ (left) and 25\% (right) of the signal rate, Bckg 1 and Bckg 2 hypotheses respectively. The result assumes $100 \%$ detector efficiency and one tonne-year exposure. Bottom graphs: same as the top graphs but for $1 \mathrm{keV}$ threshold instead. For the CE $\nu \mathrm{NS}$ analysis, these are the "experimental" signals we have assumed. As can be seen, results are rather sensitive to threshold choices. 
neutrino flavors on equal footing. For electron recoils the situation is different since electron neutrinos are subject as well to charged current processes, while the other flavors do not. Fluxes, therefore, should be weighted by the neutrino oscillation survival probability $P_{e e}$, which proceeds from an average over neutrino trajectory, including all neutrino fluxes $d \Phi / d E_{\nu}$ (pp and CNO cycles) and involving neutrino production distributions as predicted by the standard solar model (see e.g. [15]). For its calculation we have employed those given by the BS05 model [23] and the neutrino oscillation parameters best-fit-point values in [61]. Inclusion of neutrino magnetic moments can involve neutrino oscillation probabilities too, depending on whether the new couplings are or not flavor dependent.

For thresholds above $\sim 0.1 \mathrm{keV} \mathrm{CE} \nu \mathrm{NS}$ is sensitive only to the ${ }^{8} \mathrm{~B}$ neutrino flux (the hep flux is too suppressed to give a sizable signal). Neutrino-electron elastic scattering instead is sensitive to all solar neutrino fluxes, and so it is dominated by pp neutrinos. Contribution form other fluxes is small, with the main contribution given by the $0.86 \mathrm{MeV}$ ${ }^{7}$ Be line (see e.g. [62]). We write then the recoil spectra as follows

$$
\begin{aligned}
\frac{d R_{\nu-N}}{d E_{r}} & =N_{N} \int_{E_{\min }}^{E_{\max }} \frac{d \Phi_{8 \mathrm{~B}}}{d E_{\nu}}\left[\frac{d \sigma_{\nu-N}}{d E_{r}}+\frac{d \sigma_{\nu}}{d E_{r}}\right] d E_{\nu}, \\
\frac{d R_{\nu-e}}{d E_{r}} & =N_{e} \int_{E_{\min }}^{E_{\max }} \sum_{\alpha} \frac{d \Phi_{\alpha}}{d E_{\nu}}\left[P_{e e} \frac{d \sigma_{\nu-e}}{d E_{r}}+\left(1-P_{e e}\right) \frac{d \sigma_{\nu_{a}}}{d E_{r}}+\frac{d \sigma_{\nu}}{d E_{r}}\right] d E_{\nu} .
\end{aligned}
$$

Here $N_{N}$ and $N_{e}$ refer to the number of nuclei and electrons in the detector, $E_{\nu}^{\min }=$ $\sqrt{m_{N} E_{r} / 2}$ for $\mathrm{CE} \nu \mathrm{NS}$ and $E_{\nu}^{\min }=\left[E_{r}+\left(E_{r}^{2}+2 E_{r} m_{e}\right)^{1 / 2}\right] / 2$ for $\nu-e$ scattering. Index $\alpha$ runs over pp, ${ }^{8} \mathrm{~B}$, hep, ${ }^{7} \mathrm{Be}$, pep ${ }^{13} \mathrm{~N},{ }^{15} \mathrm{O}$ and ${ }^{17} \mathrm{~F}$. $E_{\nu}^{\max }$ is determined by the kinematic tail of the corresponding flux as displayed in table 1.

\section{Sensitivity to neutrino magnetic moments}

Xenon multi-ton scale DM detectors rely on photon (scintillation) and electron (ionization) signals $[3,5]$. Photons are detected through photosensors that produce a prompt S1 signal. Electrons, instead, are drifted upwards with the aid of an electric field, resulting in a delayed S2 signal. S1 and S2 signals in turn allow the reconstruction of the radial position and depth of a given interaction, together with the energy reconstruction of an event. Their ratio, $\mathrm{S} 2 / \mathrm{S} 1$, provides a way to descriminate between electron and nuclear recoils. Moreover, the dual-phase technology, allows to get more information on the S2 signal improving the resolution power of these detectors. The combination of these features provides a powerful tool for event selection over background and this will eventually enable the reconstruction of new physics signals, if any, through the discrimination of electronic and nuclear signatures.

To assess the sensitivity of direct detection experiments to neutrino magnetic moments we use a spectral chi-square test assuming various detector configurations as follows. One, ten and forty tonne active volume sizes, $0.3 \mathrm{keV}$ and $1 \mathrm{keV}$ thresholds. For CE $\nu \mathrm{NS}$ we adopt two background hypotheses, $68 \%$ and $25 \%$ of the signal rate. For $\nu-e$ elastic scattering instead we use the expected backgrounds at XENON1T, XENONnT and DARWIN reported in refs. [2, 22, 28]. They include material radioactivity, double beta decays of ${ }^{136} \mathrm{Xe}$ and ${ }^{124} \mathrm{Xe}$ decays via double electron capture, among others. Although we take 


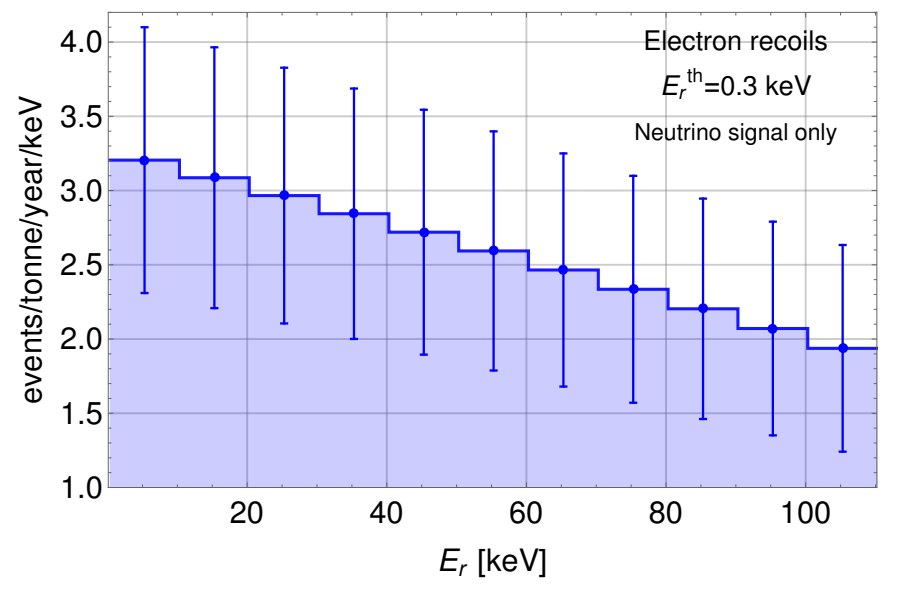

Figure 2. Neutrino-electron signal for a $0.3 \mathrm{keV}$ threshold with error bars amounting to $68 \%$ of the signal rate. The calculation assumes 100\% detector efficiency and a one tonne-year exposure. Results are rather insensitive to threshold shifts $(0.3 \mathrm{keV}$ to $1 \mathrm{keV})$.

these assumptions as representative of XENON1T, XENONnT and DARWIN, their main motivation is that of comparing the impact of different active volumes as well as different thresholds and backgrounds in the reach of direct detection experiments to neutrino electromagnetic properties.

Under these assumptions we first calculate the signals that define our toy experiments for both $\mathrm{CE} \nu \mathrm{NS}$ and $\nu-e$ elastic scattering, considered in all cases as the SM prediction $\left(\mu_{\nu_{\mathrm{eff}}}=0\right)$. For $\mathrm{CE} \nu \mathrm{NS}$, recoil energies are taken up to the ${ }^{8} \mathrm{~B}$ kinematic threshold, $\sim 4.5 \mathrm{keV}$. For $\nu-e$ scattering the pp-induced signal extends up to $264 \mathrm{keV}$ (see table 1). However, we consider recoil energies only up to $105 \mathrm{keV}$, point at which the signal drops. Covering up to the kinematic limit does not have a substantial impact in our results. The resulting toy experiments signals are shown in figure 1 for $\mathrm{CE} \nu \mathrm{NS}$ and in figure 2 for $\nu-e$ scattering. Note that we have only shown signals at different thresholds for the case of $\mathrm{CE} \nu \mathrm{NS}$. We found that for $\nu-e$ scattering, changing the threshold from $0.3 \mathrm{keV}$ to $1 \mathrm{keV}$ has a negligible effect, which means that toy experiments for any of those thresholds produce, in practice, the same signal. The reason is justified by the fact that the $0.7 \mathrm{keV}$ shift in energy threshold for $\nu-e$ scattering in the region of interest, reduces the energy range by $0.7 \%$, while for $\mathrm{CE} \nu \mathrm{NS}$ by $15 \%$.

We define our binned chi-square test according to

$$
\chi^{2}=\sum_{i} \frac{1}{\sigma_{i}^{2}}\left(\left.\frac{d R_{i}}{d E_{r}}\right|_{\mathrm{SM}}-\left.\frac{d R_{i}}{d E_{r}}\right|_{\mu_{\nu_{\mathrm{eff}}}}\right)^{2},
$$

where the recoil spectra in the second term includes both the SM and neutrino magnetic moment contributions to the signal. For our analysis we sample over $\mu_{\nu_{\mathrm{eff}}}$ from $10^{-9} \mu_{B}$ and as low as $10^{-13} \mu_{B}$ for both $\mathrm{CE} \nu \mathrm{NS}$ and $\nu-e$ scattering channels. The results of the analysis are shown in figure 3 , which display $\Delta \chi^{2}$ versus $\mu_{\nu_{\text {eff }}}$ (in $\mu_{B}$ units) calculated for the four different combinations in figure 1 . The results for different active volume sizes are shown in each plot, proving that an enhancement from 1 to 40 tonne (XENON1T to 

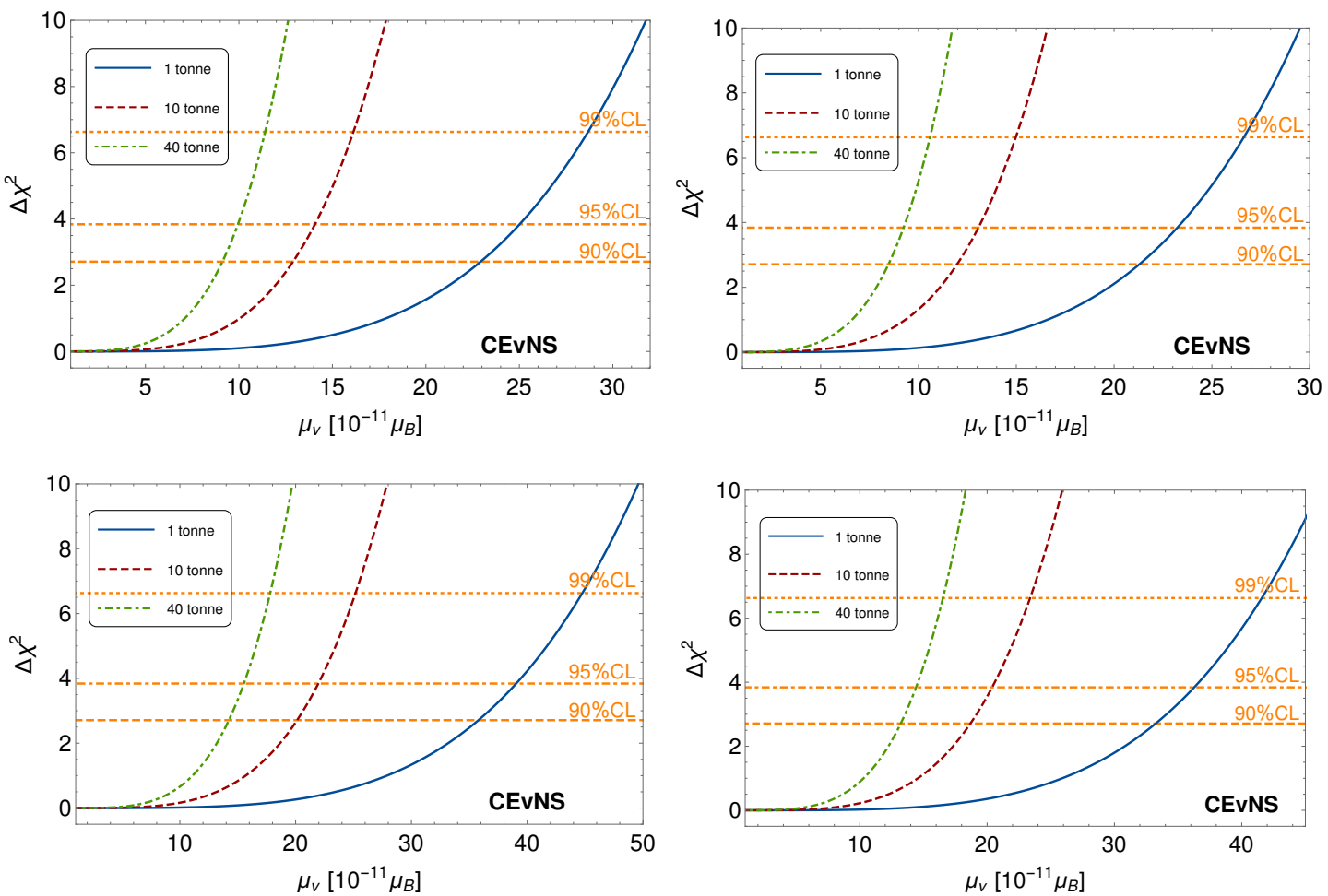

Figure 3. Top graphs: nuclear recoil sensitivities to neutrino magnetic dipole moments in a 1,10 and 40 tonne active volume detectors during a one-year data taking. The result assumes a $0.3 \mathrm{keV}$ threshold, 100\% detector efficiency and backgrounds amounting to $68 \%$ (left) and 25\% (right) of the signal rate, Bckg 1 and 2 hypotheses respectively. Bottom graphs: same as top graphs, but assuming a $1 \mathrm{keV}$ threshold. In contrast to electron recoils, results are rather sensitive to threshold performances.

DARWIN) will improve the sensitivity by a factor $\sim 2.5$ at the $90 \% \mathrm{CL}$. As can be seen this sensitivity factor enhancement is independent of threshold and background conditions.

A direct comparison of the results can be done as follows: panels in the same row share the same threshold, while those in the same column share the same background hypothesis. We can conclude that decreasing backgrounds may allow a sensitivity enhancement of order $15 \%$ at the $90 \%$ CL. Clearly, our background hypotheses are somewhat arbitrary. Changing them will quantitatively affect this conclusion, but the qualitative feature will be unchanged. Changing the threshold - as expected - has a similar impact on sensitivities, they are degraded by going from $0.3 \mathrm{keV}$ to $1 \mathrm{keV}$. Overall the best sensitivity is obtained with a 40-tonne active volume with low background and threshold, top-right graph where we can see that values as small as $8.0 \times 10^{-11} \mu_{B}$ at the $90 \% \mathrm{CL}$ can be explored. This result is remarkable since it shows that if a $0.3 \mathrm{keV}$ threshold is attainable, experiments with characteristics as those of DARWIN will be able to explore regions in parameter space rather comparable to those explored by Borexino, TEXONO and GEMMA [6, 48, 49] even in nuclear recoil measurements. Furthermore, it demonstrates that even in its nuclear recoil data sets DARWIN will be able to test regions close to those not yet ruled out by astrophysical arguments, $\left.\mu_{\nu}\right|_{\text {astro }} \lesssim 3 \times 10^{-12} \mu_{B}$ [55]. If such threshold is not achievable, 


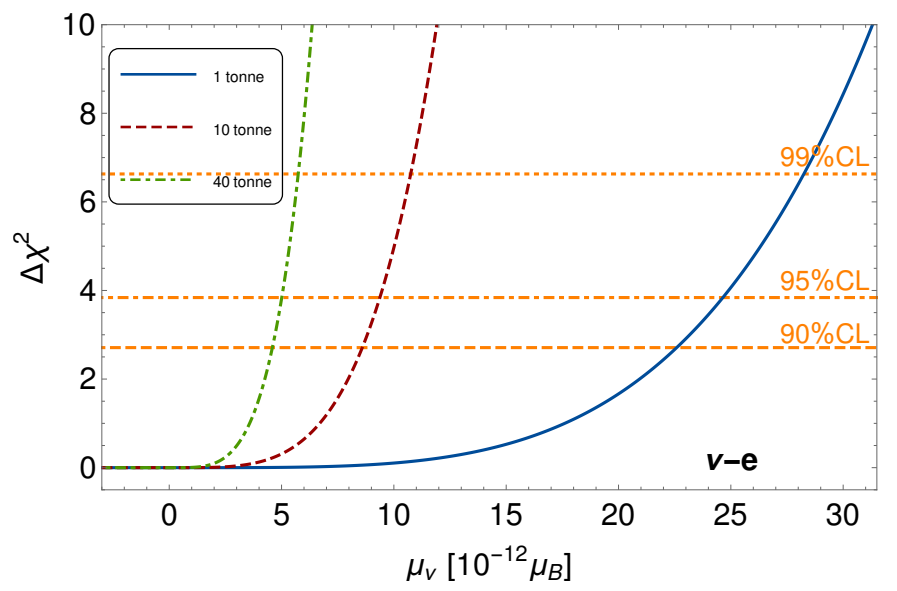

Figure 4. Electron recoil sensitivities to neutrino magnetic dipole moments in 1, 10 and 40 tonne active volume detectors during a one-year data taking. The result assumes a $0.3 \mathrm{keV}$ threshold, $100 \%$ detector efficiency and backgrounds for XENON1T, XENONnT and DARWIN as given in refs. $[2,22,28]$. The result is rather insensitive to threshold shifts $(0.1 \mathrm{keV}$ to $1 \mathrm{keV})$.

and measurements are "limited" to $1 \mathrm{keV}$ threshold instead, with low backgrounds still sensitivities like those of Borexino, TEXONO and GEMMA will be within reach in the nuclear recoil channel as the bottom-right graph in figure 3 shows.

For the neutrino-electron scattering case sensitivities are better, as expected. Results of our analysis for this process are shown in figure 4 , which displays $\Delta \chi^{2}$ versus $\mu_{\nu_{\mathrm{eff}}}$. They have been obtained by using the toy experiment signal shown in figure 2 , and by sampling over $\mu_{\nu_{\mathrm{eff}}}$ in the same range as in the CE $\nu$ NS analysis. Thanks to the low thresholds and large volume sizes, sensitivities will outpass those achieved in Borexino, TEXONO and GEMMA even in the 1-tonne detector case (representative of XENON1T). At the 90\%CL sensitivities reach values of order $2.3 \times 10^{-11} \mu_{B}$. Considering the 40 -tonne detector instead, sensitivities improve to values of about $4 \times 10^{-12} \mu_{B}$ at the $90 \% \mathrm{CL}$.

These values are of the same order and can become more competitive than those derived from astrophysical arguments, which then brings the question of the sensitivities that could be reached with other detector configurations. This question is particularly relevant in the light of existing theoretical bounds derived using effective theories or renormalizable models, which lead to values of about $10^{-14} \mu_{B}$ [63-69]. If one takes the bound from astrophysical arguments at face value, ${ }^{3}$ the region of interest then spans roughly two orders of magnitude.

To determine the degree at which the full region can be covered we have calculated the sensitivity in electron recoils that could be achieved in a hypothetical 200-tonne liquid xenon detector under the most favorable assumptions in ten years of data taking. We regard this case as the most optimistic one, and so it fixes the most ambitious sensitivity one could expect. The result is displayed in figure 5 , which shows $\Delta \chi^{2}$ versus $\mu_{\nu_{\mathrm{eff}}}$ for the

\footnotetext{
${ }^{3}$ Note that astrophysical bounds may be subject to substantially large uncertainties. So the lower boundary of the allowed region should be understood as somewhat fuzzy. This is arguably the approach adopted in ref. [2] when interpreting the electron excess in terms of neutrino magnetic moments.
} 
assumed configurations. The result includes as well an additional background assumption amounting to $5 \%$ of the signal rate, background 3 hypothesis. This result shows that if low thresholds are achieved in this type of detectors, the final sensitivity to neutrino magnetic dipole moments will be of order $10^{-12} \mu_{B}$ with little dependence on background. Under the background 2 hypothesis the best sensitivity that can be achieved is about $1.7 \times 10^{-12} \mu_{B}$ at the $90 \% \mathrm{CL}$, while with the background 3 hypothesis this value improves to $1.6 \times 10^{-12} \mu_{B}$ at the $90 \%$ CL. Finally, we have checked whether reducing statistical uncertainties could allow further improvements of these sensitivities. Assuming the background 3 hypothesis and reducing $\sigma_{a}$ in eq. (3.1) by a 0.1 factor we have found that sensitivities could improve close to values of order $10^{-13} \mu_{B}$.

In summary one can fairly say that the full region of interest cannot be covered, but perhaps a reasonable fraction of it could. Whether this is the case will largely depend on the size of statistical uncertainties. If they are substantially reduced, these detectors could eventually test regions of parameter space where non-zero neutrino magnetic moments could induce sizable signals.

\section{Conclusions}

With large fiducial volumes DM direct detection experiments are sensitive to solar neutrinos fluxes. Indeed, the statistics in both nuclear and electron recoils is expected to be large. This is the case, for instance, in XENON1T which has already collected a substantial number of events in both channels $[1,2]$. Motivated by the large statistics expected, in this paper we have studied the sensitivity of those measurements to neutrino magnetic dipole moments. We have considered different detector configurations, which although rather generic are representative of XENON1T, XENONnT and DARWIN. By generating toy experiments signals given by the SM prediction plus two background hypotheses for nuclear recoils (68\% and $25 \%$ of the signal rate) and actual background for electron recoils, we have done a chi-square test analysis to determine the reach these detectors would have.

In the case of $\mathrm{CE} \nu \mathrm{NS}$ we have found that sensitivities can be comparable to those reached by neutrino-electron elastic scattering dedicated experiments such as TEXONO, Borexino and GEMMA [6, 48, 49]. The best sensitivity can be achieved with the 40-tonne detector, with a $0.3 \mathrm{keV}$ threshold and low background. In one year of data taking such detector could explore regions in parameter space down to values of order $8.0 \times 10^{-11} \mu_{B}$ at the $90 \% \mathrm{CL}$. The 1-tonne detector operating with the same threshold and low background as well could achieve values of about $21.5 \times 10^{-11} \mu_{B}$ at the $90 \%$ CL. These sensitivities can be certainly improved with larger data taking times, but even assuming only one year is already sufficient to make nuclear recoil measurements competitive with current limits.

Sensitivities with neutrino-electron elastic scattering are better. Furthermore, they are rather insensitive to recoil thresholds. Shifting from $0.3 \mathrm{keV}$ to $1 \mathrm{keV}$ changes the events/tonne/year rate in less than $1 \%$. In the ideal case of a 40-tonne detector with a $0.3 \mathrm{keV}$ threshold, regions with values as small as $\sim 4.0 \times 10^{-12} \mu_{B}$ at the $90 \% \mathrm{CL}$ could be explored. This means that using electron recoil measurements these detectors can explore regions of parameter space not yet ruled out by astrophysical arguments. We have found 


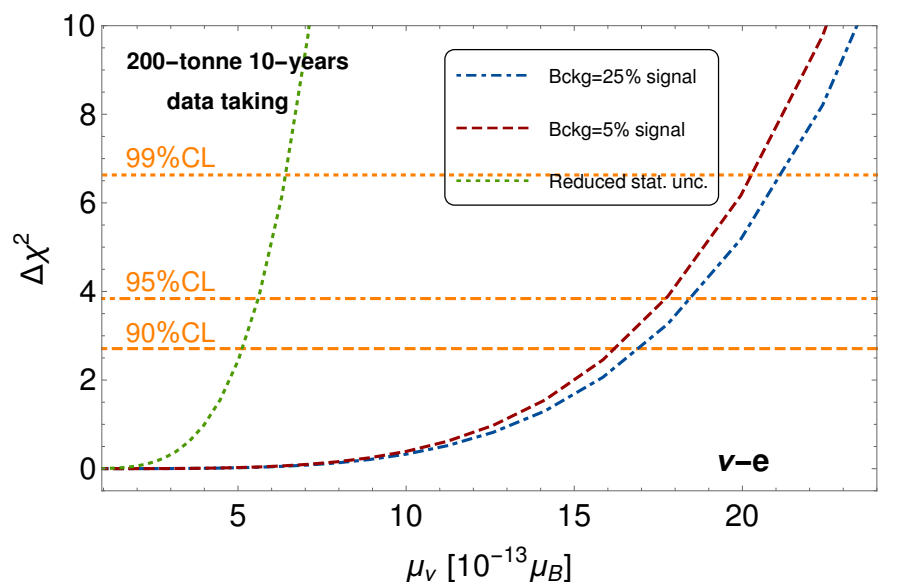

Figure 5. Electron recoil sensitivities to neutrino magnetic dipole moments in a 200-tonne liquid xenon detector under the background 2 and background 3 hypotheses $(25 \%$ and $5 \%$ of the signal rate) assuming $100 \%$ detector efficiency. Results from an analysis with a reduced statistical uncertainty are given by the dotted green curve. The result is obtained for 10 years of data taking.

that even the 1-tonne detector might be able to reach values of order $2.3 \times 10^{-11} \mu_{B}$ at the $90 \%$ CL in only one year of data taking. Note that this result is inline with the neutrino magnetic hypothesis considered by XENON1T in its electron excess analysis [2]. These results show that searches for neutrino magnetic signals are already dominated by this type of detectors and will keep being so in the future.

Finally, we have quantified the degree at which these detectors could cover the $10^{-14} \mu_{B}-10^{-12} \mu_{B}$ region with increasing data taking. To do so we calculated sensitivities for a hypothetical 200-tonne detector under two background hypotheses, $25 \%$ and $5 \%$ of the signal rate and 10 years of data taking. Our findings show that under these - somewhat extreme - conditions, sensitivities can reach values of order $1.7 \times 10^{-12} \mu_{B}$ $\left(1.6 \times 10^{-12} \mu_{B}\right)$ at the $90 \%$ CL for the background 2 (background 3$)$ hypothesis. Covering the full region of interest seems unlikely, but a reasonable fraction is potentially testable if statistical uncertainties can be further suppressed. These detectors thus have a chance to eventually observe neutrino magnetic moment induced signals.

\section{Acknowledgments}

We thank Kaixuan Ni for very useful comments on backgrounds as well as for comments on the manuscript. Jelle Aalbers for providing us details of the data in ref. [1] and Dimitris Papoulias for suggestions. DAS and RB are supported by the grant "Unraveling new physics in the high-intensity and high-energy frontiers", Fondecyt No 1171136. OGM and GSG have been supported by CONACyT through grant A1-S-23238.

Open Access. This article is distributed under the terms of the Creative Commons Attribution License (CC-BY 4.0), which permits any use, distribution and reproduction in any medium, provided the original author(s) and source are credited. 


\section{References}

[1] XENON collaboration, Light dark matter search with ionization signals in XENON1T, Phys. Rev. Lett. 123 (2019) 251801 [arXiv: 1907.11485] [INSPIRE].

[2] XENON collaboration, Excess electronic recoil events in XENON1T, Phys. Rev. D 102 (2020) 072004 [arXiv:2006.09721] [INSPIRE].

[3] XENON collaboration, Physics reach of the XENON1T dark matter experiment, JCAP 04 (2016) 027 [arXiv: 1512.07501] [INSPIRE].

[4] LUX-ZEPLIN collaboration, Projected WIMP sensitivity of the LUX-ZEPLIN dark matter experiment, Phys. Rev. D 101 (2020) 052002 [arXiv: 1802.06039] [InSPIRE].

[5] DARWIN collaboration, DARWIN: towards the ultimate dark matter detector, JCAP 11 (2016) 017 [arXiv:1606.07001] [INSPIRE].

[6] TEXONO collaboration, Measurement of $\bar{\nu}_{e}$-e scattering cross-section with a CsI(Tl) scintillating crystal array at the Kuo-Sheng nuclear power reactor, Phys. Rev. D 81 (2010) 072001 [arXiv:0911.1597] [INSPIRE].

[7] JUNO collaboration, Neutrino physics with JUNO, J. Phys. G 43 (2016) 030401 [arXiv: 1507.05613] [INSPIRE].

[8] DUNE collaboration, Long-Baseline Neutrino Facility (LBNF) and Deep Underground Neutrino Experiment (DUNE): conceptual design report, volume 2: the physics program for DUNE at LBNF, arXiv:1512.06148 [INSPIRE].

[9] R. Strauss et al., The $\nu$-cleus experiment: a Gram-scale fiducial-volume cryogenic detector for the first detection of coherent neutrino-nucleus scattering, Eur. Phys. J. C 77 (2017) 506 [arXiv: 1704.04320] [INSPIRE].

[10] J. Hakenmüller et al., Neutron-induced background in the CONUS experiment, Eur. Phys. J. C 79 (2019) 699 [arXiv:1903.09269] [INSPIRE].

[11] CONNIE collaboration, Exploring low-energy neutrino physics with the Coherent Neutrino Nucleus Interaction Experiment, Phys. Rev. D 100 (2019) 092005 [arXiv:1906.02200] [INSPIRE].

[12] L. Baudis, A. Ferella, A. Kish, A. Manalaysay, T. Marrodan Undagoitia and M. Schumann, Neutrino physics with multi-ton scale liquid xenon detectors, JCAP 01 (2014) 044 [arXiv:1309.7024] [INSPIRE].

[13] D.G. Cerdeño, M. Fairbairn, T. Jubb, P.A.N. Machado, A.C. Vincent and C. Bøehm, Physics from solar neutrinos in dark matter direct detection experiments, JHEP 05 (2016) 118 [Erratum ibid. 09 (2016) 048] [arXiv:1604.01025] [INSPIRE].

[14] B. Dutta, S. Liao, L.E. Strigari and J.W. Walker, Non-standard interactions of solar neutrinos in dark matter experiments, Phys. Lett. B 773 (2017) 242 [arXiv:1705.00661] [INSPIRE].

[15] D. Aristizabal Sierra, N. Rojas and M.H.G. Tytgat, Neutrino non-standard interactions and dark matter searches with multi-ton scale detectors, JHEP 03 (2018) 197 [arXiv: 1712.09667] [INSPIRE].

[16] M.C. Gonzalez-Garcia, M. Maltoni, Y.F. Perez-Gonzalez and R. Zukanovich Funchal, Neutrino discovery limit of dark matter direct detection experiments in the presence of non-standard interactions, JHEP 07 (2018) 019 [arXiv: 1803.03650] [INSPIRE]. 
[17] G.-Y. Huang and S. Zhou, Constraining neutrino lifetimes and magnetic moments via solar neutrinos in the large xenon detectors, JCAP 02 (2019) 024 [arXiv:1810.03877] [INSPIRE].

[18] D. Aristizabal Sierra, B. Dutta, S. Liao and L.E. Strigari, Coherent elastic neutrino-nucleus scattering in multi-ton scale dark matter experiments: classification of vector and scalar interactions new physics signals, JHEP 12 (2019) 124 [arXiv:1910.12437] [INSPIRE].

[19] D.K. Papoulias, R. Sahu, T.S. Kosmas, V.K.B. Kota and B. Nayak, Novel neutrino-floor and dark matter searches with deformed shell model calculations, Adv. High Energy Phys. 2018 (2018) 6031362 [arXiv: 1804.11319] [INSPIRE].

[20] C.-C. Hsieh et al., Discovery potential of multiton xenon detectors in neutrino electromagnetic properties, Phys. Rev. D 100 (2019) 073001 [arXiv:1903.06085] [INSPIRE].

[21] J.L. Newstead, L.E. Strigari and R.F. Lang, Detecting CNO solar neutrinos in next-generation xenon dark matter experiments, Phys. Rev. D 99 (2019) 043006 [arXiv: 1807.07169] [INSPIRE].

[22] DARWIN collaboration, Solar neutrino detection sensitivity in DARWIN via electron scattering, Eur. Phys. J. C 80 (2020) 1133 [arXiv:2006.03114] [INSPIRE].

[23] J.N. Bahcall, A.M. Serenelli and S. Basu, New solar opacities, abundances, helioseismology, and neutrino fluxes, Astrophys. J. Lett. 621 (2005) L85 [astro-ph/0412440] [INSPIRE].

[24] D. Aristizabal Sierra, V. De Romeri and N. Rojas, CP violating effects in coherent elastic neutrino-nucleus scattering processes, JHEP 09 (2019) 069 [arXiv:1906.01156] [INSPIRE].

[25] L.B. Okun, Limits of electrodynamics: paraphotons?, Sov. Phys. JETP 56 (1982) 502 [Zh. Eksp. Teor. Fiz. 83 (1982) 892] [INSPIRE].

[26] B. Holdom, Two U(1)'s and epsilon charge shifts, Phys. Lett. B 166 (1986) 196 [InSPIRE].

[27] B. Lenardo et al., Measurement of the ionization yield from nuclear recoils in liquid xenon between $0.3-6 \mathrm{keV}$ with single-ionization-electron sensitivity, arXiv: 1908.00518 [INSPIRE].

[28] XENON collaboration, Projected WIMP sensitivity of the XENONnT dark matter experiment, JCAP 11 (2020) 031 [arXiv:2007.08796] [INSPIRE].

[29] D.Z. Freedman, Coherent neutrino nucleus scattering as a probe of the weak neutral current, Phys. Rev. D 9 (1974) 1389 [InSPIRE].

[30] D.Z. Freedman, D.N. Schramm and D.L. Tubbs, The weak neutral current and its effects in stellar collapse, Ann. Rev. Nucl. Part. Sci. 27 (1977) 167 [InSPIRE].

[31] J.L. Newstead, R.F. Lang and L.E. Strigari, Atmospheric neutrinos in a next-generation xenon dark matter experiment, arXiv:2002.08566 [INSPIRE].

[32] R.F. Lang, C. McCabe, S. Reichard, M. Selvi and I. Tamborra, Supernova neutrino physics with xenon dark matter detectors: A timely perspective, Phys. Rev. D 94 (2016) 103009 [arXiv: 1606.09243] [INSPIRE].

[33] Particle Data Group collaboration, Review of particle physics, Chin. Phys. C 40 (2016) 100001.

[34] D. Aristizabal Sierra, J. Liao and D. Marfatia, Impact of form factor uncertainties on interpretations of coherent elastic neutrino-nucleus scattering data, JHEP 06 (2019) 141 [arXiv: 1902.07398] [INSPIRE].

[35] P. Vogel and J. Engel, Neutrino electromagnetic form-factors, Phys. Rev. D 39 (1989) 3378 [INSPIRE]. 
[36] K. Fujikawa and R. Shrock, The magnetic moment of a massive neutrino and neutrino spin rotation, Phys. Rev. Lett. 45 (1980) 963 [INSPIRE].

[37] J. Schechter and J.W.F. Valle, Majorana neutrinos and magnetic fields, Phys. Rev. D 24 (1981) 1883 [Erratum ibid. 25 (1982) 283] [INSPIRE].

[38] P.B. Pal and L. Wolfenstein, Radiative decays of massive neutrinos, Phys. Rev. D 25 (1982) 766 [INSPIRE].

[39] B. Kayser, Majorana neutrinos and their electromagnetic properties, Phys. Rev. D 26 (1982) 1662 [INSPIRE].

[40] J.F. Nieves, Electromagnetic properties of Majorana neutrinos, Phys. Rev. D 26 (1982) 3152 [INSPIRE].

[41] R.E. Shrock, Electromagnetic properties and decays of Dirac and Majorana neutrinos in a general class of gauge theories, Nucl. Phys. B 206 (1982) 359 [inSPIRE].

[42] B.C. Canas, O.G. Miranda, A. Parada, M. Tortola and J.W.F. Valle, Updating neutrino magnetic moment constraints, Phys. Lett. B 753 (2016) 191 [Addendum ibid. 757 (2016) 568] [arXiv: 1510.01684] [INSPIRE].

[43] F. Della Valle et al., The PVLAS experiment: measuring vacuum magnetic birefringence and dichroism with a birefringent Fabry-Perot cavity, Eur. Phys. J. C 76 (2016) 24 [arXiv: 1510.08052] [INSPIRE].

[44] R. Foot, G.C. Joshi, H. Lew and R.R. Volkas, Charged neutrinos?, Mod. Phys. Lett. A 5 (1990) 95 [Erratum ibid. 5 (1990) 2085] [INSPIRE].

[45] M. Hirsch, E. Nardi and D. Restrepo, Bounds on the tau and muon neutrino vector and axial vector charge radius, Phys. Rev. D 67 (2003) 033005 [hep-ph/0210137] [INSPIRE].

[46] MUNU collaboration, Final results on the neutrino magnetic moment from the MUNU experiment, Phys. Lett. B $\mathbf{6 1 5}$ (2005) 153 [hep-ex/0502037] [INSPIRE].

[47] Super-Kamiokande collaboration, Limits on the neutrino magnetic moment using 1496 days of Super-Kamiokande-I solar neutrino data, Phys. Rev. Lett. 93 (2004) 021802 [hep-ex/0402015] [INSPIRE].

[48] A.G. Beda et al., Upper limit on the neutrino magnetic moment from three years of data from the GEMMA spectrometer, arXiv:1005.2736 [INSPIRE].

[49] Borexino collaboration, Limiting neutrino magnetic moments with Borexino Phase-II solar neutrino data, Phys. Rev. D 96 (2017) 091103 [arXiv:1707.09355] [INSPIRE].

[50] J.A. Grifols and E. Masso, Bound on the neutrino charge radius from primordial nucleosynthesis, Mod. Phys. Lett. A 2 (1987) 205 [INSPIRE].

[51] A.I. Studenikin and I. Tokarev, Millicharged neutrino with anomalous magnetic moment in rotating magnetized matter, Nucl. Phys. B 884 (2014) 396 [arXiv:1209.3245] [INSPIRE].

[52] G. Barbiellini and G. Cocconi, Electric charge of the neutrinos from SN1987A, Nature 329 (1987) 21 [INSPIRE].

[53] J.A. Grifols and E. Masso, Charge radius of the neutrino: a limit from SN1987A, Phys. Rev. D 40 (1989) 3819 [INSPIRE].

[54] G.G. Raffelt, Astrophysical methods to constrain axions and other novel particle phenomena, Phys. Rept. 198 (1990) 1 [INSPIRE]. 
[55] G.G. Raffelt, New bound on neutrino dipole moments from globular cluster stars, Phys. Rev. Lett. 64 (1990) 2856 [INSPIRE].

[56] C. Giunti and A. Studenikin, Neutrino electromagnetic interactions: a window to new physics, Rev. Mod. Phys. 87 (2015) 531 [arXiv:1403.6344] [INSPIRE].

[57] V. Brdar, A. Greljo, J. Kopp and T. Opferkuch, The neutrino magnetic moment portal: cosmology, astrophysics, and direct detection, arXiv:2007.15563 [INSPIRE].

[58] A.N. Khan, $\sin ^{2} \theta_{W}$ estimate and neutrino electromagnetic properties from low-energy solar data, J. Phys. G 46 (2019) 035005 [arXiv: 1709. 02930] [inSPIRE].

[59] M. Lindner, F.S. Queiroz, W. Rodejohann and X.-J. Xu, Neutrino-electron scattering: general constraints on $Z^{\prime}$ and dark photon models, JHEP 05 (2018) 098 [arXiv:1803.00060] [INSPIRE].

[60] J.F. Beacom and P. Vogel, Neutrino magnetic moments, flavor mixing, and the Super-Kamiokande solar data, Phys. Rev. Lett. 83 (1999) 5222 [hep-ph/9907383] [InSPIRE].

[61] P.F. de Salas, D.V. Forero, C.A. Ternes, M. Tortola and J.W.F. Valle, Status of neutrino oscillations 2018: $3 \sigma$ hint for normal mass ordering and improved CP sensitivity, Phys. Lett. B 782 (2018) 633 [arXiv:1708.01186] [INSPIRE].

[62] D. Aristizabal Sierra, V. De Romeri, L.J. Flores and D.K. Papoulias, Light vector mediators facing XENON1T data, Phys. Lett. B 809 (2020) 135681 [arXiv:2006.12457] [INSPIRE].

[63] R. Shrock, Decay $l_{0} \rightarrow \nu_{\ell} \gamma$ in gauge theories of weak and electromagnetic interactions, Phys. Rev. D 9 (1974) 743 [inSPIRE].

[64] K.S. Babu and R.N. Mohapatra, Supersymmetry and large transition magnetic moment of the neutrino, Phys. Rev. Lett. 64 (1990) 1705 [InSPIRE].

[65] S.M. Barr, E.M. Freire and A. Zee, A mechanism for large neutrino magnetic moments, Phys. Rev. Lett. 65 (1990) 2626 [INSPIRE].

[66] N.F. Bell, V. Cirigliano, M.J. Ramsey-Musolf, P. Vogel and M.B. Wise, How magnetic is the Dirac neutrino?, Phys. Rev. Lett. 95 (2005) 151802 [hep-ph/0504134] [INSPIRE].

[67] S. Davidson, M. Gorbahn and A. Santamaria, From transition magnetic moments to Majorana neutrino masses, Phys. Lett. B 626 (2005) 151 [hep-ph/0506085] [INSPIRE].

[68] N.F. Bell, M. Gorchtein, M.J. Ramsey-Musolf, P. Vogel and P. Wang, Model independent bounds on magnetic moments of Majorana neutrinos, Phys. Lett. B 642 (2006) 377 [hep-ph/0606248] [INSPIRE].

[69] K.S. Babu, S. Jana and M. Lindner, Large neutrino magnetic moments in the light of recent experiments, JHEP 10 (2020) 040 [arXiv:2007.04291] [INSPIRE]. 